\title{
Survey on Privacy-Preserving Detection of Sensitive Data Exposure
}

\author{
Madhavi R Suryawanshi ${ }^{1}$, Sarita A Patil ${ }^{2}$ \\ ${ }^{1} \mathrm{ME}$ (CE), GHRCEM, Wagholi, Pune, India \\ ${ }^{2}$ Guide and Professor, GHRCEM, Wagholi, Pune, India
}

\begin{abstract}
Measurements from security firms, research foundations what's more, government associations demonstrate that the quantity of information hole occasions have developed quickly as of late. Here human mix-ups are one of the fundamental drivers of information misfortune. Such a methodology as a rule requires the identification operation to be directed in mystery. In this paper, we introduce a privacy preserving information spill location (DLD) answer for fathom the issue where an extraordinary arrangement of delicate information summaries is utilized as a part of identification. The upside of our system is that it empowers the information proprietor to securely appoint the discovery operation to a semi honest supplier without uncovering the touchy information to the supplier. We portray how Internet administration suppliers can offer their clients DLD as an extra administration with solid protection ensures. Here system can bolster exact recognition with little number of false cautions under different information spill situations.
\end{abstract}

Keywords: Data leak, network security, privacy, collection intersection.

\section{Introduction}

As indicated by a report from Risk Based Security (RBS), the quantity of released touchy information records has expanded drastically amid the last couple of years, i.e., from 412 million in 2012 to 822 million in 2013. Purposely arranged assaults, incidental holes (e.g., sending private messages to unclassified email records), and human missteps (e.g., relegating the off-base benefit) lead to the vast majority of the information spill episode. Distinguishing and forestalling information holes requires an arrangement of integral arrangements, which may incorporate information spill recognition, information control stealthy malware recognition, and strategy requirement. Network information spill location (DLD) normally performs profound bundle review (DPI) and looks for any events of delicate information designs. DPI is a method to examine payloads of IP/TCP bundles for reviewing application layer information, e.g., HTTP header/content. Alarms are activated when the measure of touchy information found in movement passes a limit. The recognition framework can be conveyed on a switch or incorporated into existing system interruption recognition frameworks (NIDS).Direct acknowledge of information break discovery require the plaintext touchy information. Nonetheless, this prerequisite is undesirable, as it may undermine the classification of the touchy data. On the off chance that a discovery framework is traded off, at that point it may uncover the plaintext touchy information (in memory). Furthermore, the information proprietor may need to outsource the information spill discovery to suppliers, yet may be unwilling to uncover the plaintext delicate information to them. In this manner, one needs new information spill discovery arrangements that permit the suppliers to sweep content for holes without taking in the delicate data. Direct acknowledge of information break discovery require the plaintext touchy information. Nonetheless, this prerequisite is undesirable, as it may undermine the classification of the touchy data. On the off chance that a discovery framework is traded off, at that point it may uncover the plaintext touchy information (in memory). Furthermore, the information proprietor may need to outsource the information spill discovery to suppliers, yet may be unwilling to uncover the plaintext delicate information to them. In this manner, one needs new information spill discovery arrangements that permit the suppliers to sweep content for holes without taking in the delicate data.

\section{Related Work}

In previous system, they tend to propose a data-leak detection answer which can be outsourced and be deployed during semi honest detection surroundings. Also design, implement, and evaluate our fuzzy fingerprint technique that enhances knowledge privacy throughout data-leak detection operations. Their approach is based on a quick and sensible unidirectional computation on the sensitive knowledge (SSN records, classified documents, sensitive emails, etc.). It permits the information owner to firmly delegate the content-inspection task to DLD suppliers while not exposing the sensitive knowledge. victimization our detection technique, the DLD supplier, who is shapely as associate honest-but-curious (aka semi-honest) adversary, will solely gain restricted data regarding the sensitive data from either the discharged digests, or the content being inspected. Victimization the techniques, an online service provider (ISP) will perform detection on its customers' traffic securely and supply data-leak detection as associate add-on service for its customers. In another situation, people will mark their own sensitive knowledge and raise the administrator of their native network to discover knowledge leaks for them.

In [5], present an method for quantifying data leak capability in the network traffic. Instead of trying to sense the occurrence of sensitive information an impossible task in the universal case - their goal is to calculate and limit its highest volume. They also propose the measurement algorithms for the Hypertext Transfer Protocol (HTTP), the main protocol for web browsing. The results were best for the blog

\section{Volume 4 Issue 12, December 2015}




\section{International Journal of Science and Research (IJSR) \\ ISSN (Online): 2319-7064 \\ Index Copernicus Value (2013): 6.14 | Impact Factor (2014): 5.611}

scenario because the blog website. The main advantage of this paper is that this paper insight that most network traffic is repeated or determined by external. In this system network traffic is so voluminous that manual inspection would be unreasonably expensive.

In [6], Panor proposes a framework, Panorama, for identifying and break down malware by catching this fundamental trait. In this system extensive experiment, Panorama effectively identified all the malware tests and had not very few false positives. Besides, by utilizing Google Desktop as a case study, they demonstrate that their framework can precisely catch its data get to and preparing conduct, and they can confirm that it sends back delicate data to remote servers in specific settings. This system can accurately capture its information access and processing behaviour. Malicious programs spy on user's behaviour and compromise their privacy. Even software from reputable vendors, such as Google Desktop and Sony DRM. Google Desktop as a case study, they show that our system can accurately capture its information access and processing behaviour, and they can confirm that it does send back sensitive information to remote Servers in certain settings.

In [7], presents Storages Capsules, a novel methodology for securing private documents on an individual PC. Storage Capsules are encoded record containers that permit a compromised machine to safely view and alter sensitive documents without malware being able to steal information.

The framework accomplishes this objective by taking a checkpoint of the present framework state and disabling device output before permitting access a Storage Capsule. Composes to the Storage Capsule are then sent to a trusted module. The trusted module declassifies the Storage Capsule by re-encrypting its contents, and exports it for storage in a low-integrity environment. Storage Capsules are encrypted file containers that allow a compromised machine to securely view and edit sensitive files without malware being able to steal confidential data. The main limitation Suffer huge losses if private data falls into the wrong hands. One of the primary threats to confidentiality is malicious software on personal computers.

In [8], presents Aquifer as an arrangement structure and framework for counteracting accidental data exposure in advanced working frameworks. In Aquifer, application engineers define secrecy confinements that ensure the whole client interface work process characterizing the client task Aquifer provides protection beyond simple permission checks and allows applications to retain control of data even after it is shared. Using file permission also avoids ambiguous read-write file open masks, as well as properly propagating labels when the workflow label changes between file open and file write.

\section{Architectural View}

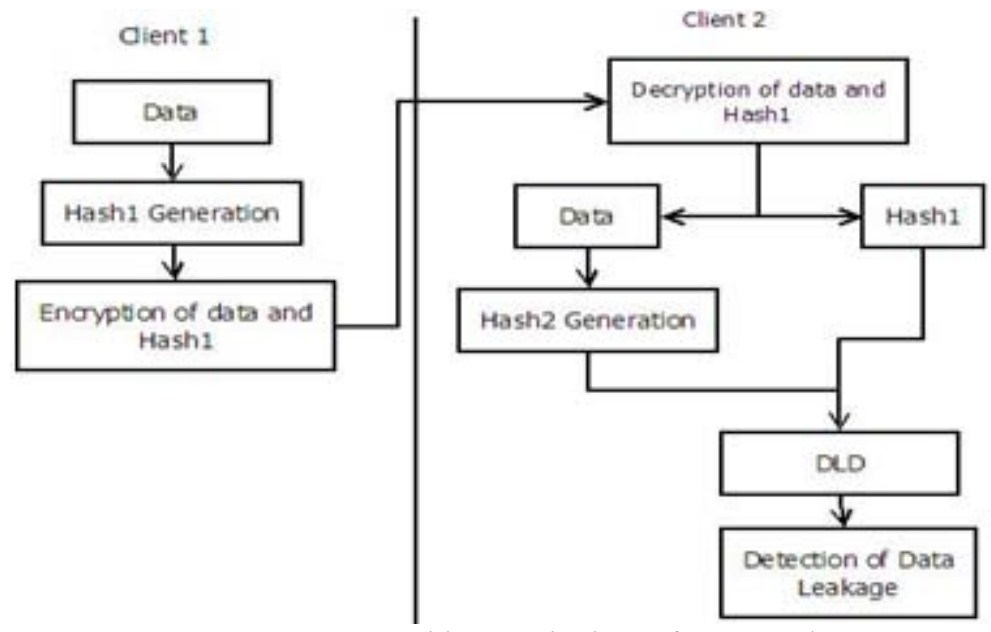

Figure 3.1: Architectural View of Proposed System

\section{Proposed Method}

\subsection{Fuzzy Fingerprint Method And Protocol}

4.1.1 Shingles and Fingerprints

4.1.2 Operations in Our Protocol

4.1.3 Extensions

\subsection{Operations in Our Protocol}

\subsubsection{Preprocess}

This operation is run by the data owner on each piece of sensitive data. a) The data owner chooses four public parameters (q, $p(x)$, $\mathrm{pd}, \mathrm{M}) . \mathrm{q}$ is the length of a shingle. $\mathrm{p}(\mathrm{x})$, is an irreducible polynomial (degree of $\mathrm{p} \mathrm{f}+1$ ) used in Rabin fingerprint

b) The data owner computes $\mathrm{S}$, which is the set of all Rabin fingerprints of the piece of sensitive data

c) The data owner transforms each fingerprint $\mathrm{f} \in \mathrm{S}$ into a fuzzy fingerprint $\mathrm{f} *$ with randomized bits (specified by the mask $M$ ). $\mathrm{f} *=((\mathrm{NOT} \mathrm{M})$ AND $\mathrm{f})$ XOR $\mathrm{f}$

\subsubsection{Release}

This operation is run by the data owner. The fuzzy fingerprint set $\mathrm{S} *$ obtained by PREPROCESS is released to the DLD provider for use in the detection, along with the public parameters $(\mathrm{q}, \mathrm{p}(\mathrm{x}), \mathrm{pd}, \mathrm{M})$. 


\section{International Journal of Science and Research (IJSR) \\ ISSN (Online): 2319-7064}

Index Copernicus Value (2013): 6.14 | Impact Factor (2014): 5.611

\subsubsection{Monitor}

This operation is run by the DLD provider. The DLD provider monitors the network traffic $\mathrm{T}$ from the data owner's organization. Each packet in $\mathrm{T}$ is collected and the payload of it is sent to the next operation as the network traffic (binary) string $\mathrm{T}^{\sim}$.

\subsubsection{Detect}

This operation is run by the DLD provider on each $\mathrm{T}^{\sim}$

\subsubsection{Report}

If DETECTION on $\mathrm{T}^{\sim}$ yields an alert, the DLD provider reports the set of detected candidate leak instances ${ }^{\wedge} \mathrm{T}$ to the data owner.

\subsubsection{Postprocess}

After receiving ${ }^{\wedge} \mathrm{T}$, the data owner test every $f \in{ }^{\wedge} \mathrm{T}$ to see whether it is in S.

\subsection{Extensions}

\subsubsection{Fingerprint Filter}

We develop this extension to use Bloom filter in the DETECT operation for efficient set intersection test.

4.3.2 Bloom filter is a well-known space-saving data structure for performing set-membership test.

4.3.3 Bloom filter in combination with Rabin fingerprint is referred to by us as the fingerprint filter.

\section{Conclusion}

We proposed fuzzy fingerprint, a privacy-preserving dataleak detection model and present its realization. Using special digests, the exposure of the sensitive data is kept to a minimum during the detection. We have conducted extensive experiments to validate the accuracy, privacy, and efficiency of our solutions. For future work, we plan to focus on designing a host-assisted mechanism for the complete data-leak detection for large-scale organizations.

\section{Future Scope}

Author plan to focus on designing a host-assisted mechanism for the complete data leak detection for largescale organizations.

\section{References}

[1] Xiaokui Shu, Danfeng Yao, Privacy-Preserving Detection of Sensitive Data Exposure", IEEE TRANSACTIONS ON INFORMATION FORENSICS AND SECURITY, VOL. 10, NO. 5, MAY 2015

[2] X. Shu and D. Yao, Đata leak detection as a service," in Proc. 8th Int.Conf. Secur. Privacy Common. Newt. 2012, pp. 222-240.

[3] Ponemon Institute. (May 2013). 2013 Cost of Data Breach Study: Global Analysis. [Online].Available:https://www4.symantec.com/mktginf owhitepaper/053013_GL_NA_WP_Ponemon-2013-
Cost-of-a-Data-Breach-Report_daiNA_cta72382.pdf, accessed Oct. 2014.

[4] Identity Finder. Discover Sensitive Data Prevent Breaches DLP Data Loss Prevention. [Online]. Available: http://www.identityfinder.com/,accessed Oct. 2014.

[5] K. Borders and A. Parkas, -Qantifying information leaks in outbound web traffic," in Proc. 30th IEEE Sump. Secure. Privacy, May 2009, pp. 129-140.

[6] H. Yin, D. Song, M. Agile, C. Kruegel, and E. Kirda, Panorama: Capturing system-wide information flow for malware detection and analysis," in Proc. 14th ACM Conf. Compute. Commun. Secur., 2007,pp. 116-127.

[7] K. Borders, E. V. Weele, B. Lau, and A. Prakash, Protecting confidential data on personal computers with storage capsules," in Proc. $18^{\text {th }}$ USENIX Secur. Symp., 2009, pp. 367-382.

[8] A. Nadkarni and W. Neck, - Peventing accidental data disclosure in modern operating systems," in Proc. 20th ACM Conf. Compute. Commun. Secur., 2013, pp. 10291042. 